\title{
NEIGHBORHOOD FIXED PENDANT VERTICES
}

BY

\author{
S. E. ANACKER AND G. N. ROBERTSON ${ }^{1}$
}

\begin{abstract}
If $x$ is pendant in $G$, then $x *$ denotes the unique vertex of $G$ adjacent to $x$. Such an $x$ is said to be neighborhood-fixed whenever $x^{*}$ is fixed by $A(G-x)$. It is shown that if $G$ is not a tree and has a pendant vertex, but no *-fixed pendant vertex, then there is a subgraph $G^{\sharp}$ of $G$ such that for some $y \in V\left(G^{\sharp}\right), O\left(A\left(G^{\#}\right)_{y}\right)$ $>t$ ! where $t$ is the maximum number of edges in a tree rooted in $G^{\#}$.
\end{abstract}

Let $G$ denote a finite connected graph without loops or multiple edges. Let $V(G)$, $E(G)$ and $A(G)$ denote respectively the vertex set, the edge set, and the automorphism group of $G$. Let $x \in V(G)$. The valency of $x$ in $G$ is denoted by $\operatorname{val}(G, x)$, and $x$ is defined to be pendant in $G$ if $\operatorname{val}(G, x)=1$. The subgraph of $G$ obtained by deleting $x$ and all edges incident with $x$ is denoted by $G-x$. If $x$ is pendant in $G$, then $x^{*}$ denotes the unique vertex of $G$ adjacent to $x$. Such an $x$ is said to be neighborhood-fixed whenever $x^{*}$ is fixed by $A(G-x)$. Neighborhood-fixed will be denoted by $*$-fixed for the remainder of the paper.

1. Tree growth number. A pruning of $G$ is a decomposition:

$$
G=G^{\sharp} \cup T_{1} \cup T_{2} \cup \cdots \cup T_{k},
$$

where $G^{\#}$ is the maximal subgraph of $G$, each vertex of which has valency $\geqslant 2$, and where $\left\{T_{i}: 1 \leqslant i \leqslant k\right\}$ is a nonempty set of disjoint nontrivial rooted trees each having only its root vertex $x_{i}$ in $G^{\sharp}$. Note that $G$ has a pruning if and only if it is not a tree and has a pendant vertex. Moreover, $G^{\#}$ is connected because $G$ is connected, and the decomposition is unique up to the order of the $T_{i}$.

In [1], Robertson and Zimmer conjectured: If $G$ is a finite graph having a pruning such that

$$
\max \left\{\left|E\left(T_{i}\right)\right|: 1 \leqslant i \leqslant k\right\}>\max \left\{\operatorname{val}\left(G^{\sharp}, x\right): x \in V\left(G^{\sharp}\right)\right\}
$$

then $G$ has $a *$-fixed pendant vertex.

There are counterexamples to the conjecture. One class of counterexamples is found among $G$ with $G^{\sharp}$ a rooted tree with triangles affixed to the pendant vertices.

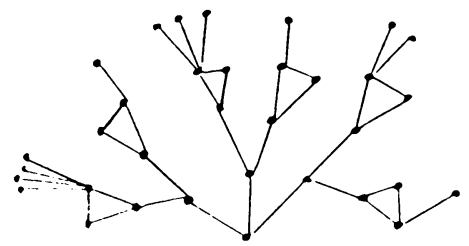

FIGURE 1

Received by the editors July 12, 1978.

1980 Mathematics Subject Classification. Primary 05C60, 05 C25.

Key words and phrases. Reducible partitions, neighborhood fixed pendant vertices.

${ }^{1}$ The work of the second author was partially supported by NSF Grant MCS 77-04926. 
Another counterexample can be constructed from Tutte's 8-cage, mentioned in [2]. Let $G^{\sharp}$ be the 8-cage. There exists a subset $\left\{x_{0}, x_{1}, x_{2}, x_{3}, x_{4}\right\}$ of $V\left(G^{\sharp}\right)$ on which $A\left(G^{\sharp}\right)$ acts as the symmetric group $S_{5}$. Then a pruning $G=G^{\sharp} \cup T_{1} \cup T_{2}$ $\cup T_{3} \cup T_{4}$, where $T_{i}$ is an $i$-star with center the root $x_{i}$ for $1<i<4$, defines a graph $G$ with no *-fixed pendant vertex.

Let $A$ be a finite group acting on a finite set $X$. Let $Y \subseteq X$ and $x \in X$. Denote the order of $A$ by $o(A)$, the subgroup of $A$ fixing $x$ by $A_{x}$, and the subgroup of $A$ fixing $Y$ setwise by $A_{(Y)}$. The restriction of $A_{(Y)}$ to $Y$ is denoted by $A^{Y}$. Denote the group generated by elements $\varphi_{1}, \varphi_{2}, \ldots, \varphi_{k}$ by $\left\langle\varphi_{1}, \ldots, \varphi_{k}\right\rangle$ and the group generated by subgroups $H_{1}, \ldots, H_{k}$ by $\left\langle H_{1}, \ldots, H_{k}\right\rangle$.

An ordered partition $X=\cup_{i=0}^{s} X_{i}$ is reducible if $s>1, X_{s} \neq \varnothing$, and for every $x \in X_{i}, i \geqslant 1$, there exists a reducing map $\alpha \in A$ such that $(x) \alpha \in X_{i-1}$ and $\alpha$ stabilizes the blocks of the partition $X_{0} \cup \cdots \cup\left(X_{i-1} \cup x\right) \cup\left(X_{i}-x\right)$ $\cup \cdots \cup X_{s}$. Let $t(A, X)$ denote the largest $s$ such that a reducible partition exists.

Note that for $x \in X_{i}, i \geqslant 1$, there may be many maps that reduce $x$. Let $\alpha_{x}$ be one such reducing map. If $Z \subset X_{i}$, $i>1$, let $I(Z)=\left\{(z) \alpha_{z}: z \in Z\right\}$. If $H$ is a subgroup of $A$ that contains the group generated by the reducing maps $\left\{\alpha_{y}\right.$ : $y \in Y$ \} then $H$ reduces $Y$. An orbit $O$ of a subgroup $H$ of $A$ is a reducing orbit if there exists a $z \in O$ such that $\alpha_{z} \in H$.

Let $G$ be a graph with a pruning. Suppose that $G$ has no *-fixed pendant vertex. Let $T_{i}, 1 \leqslant i \leqslant k$, be the trees of the pruning of $G$. Replace each tree $T_{i}$ by a star $S_{i}$ with $\left|E\left(S_{i}\right)\right|=\left|E\left(T_{i}\right)\right|$ rooted at its center $x_{i}$ to form a new graph $G^{\prime}$ with a pruning. Then $G^{\prime}$ also has no *-fixed pendant vertex. To see this, suppose on the contrary that $x \in V\left(S_{i}\right)$ is a *-fixed pendant vertex. Then $x_{i}$ is fixed by $A\left(G^{\prime}-x_{i}\right)$. Let the weight, $w(A)$, of $A \in E\left(T_{i}\right)$ be the number of edges in the component of $\left(T_{i}\right)_{A}^{\prime}$ (formed by deleting $A$ from $T_{i}$ ), which does not contain $x_{i}$. Suppose $\left(x_{i}, A_{1}, a_{1}, A_{2}, a_{2}, \ldots, a_{k-1}, A_{k}, x\right)$ is a path in $T_{i}$ from $x_{i}$ to a pendant vertex $x$ following successive lightest possible edges. Then $x$ is a $*$-fixed pendant vertex of $G$, contrary to assumptions.

$G$ will be assumed to have a pruning such that $G^{\#}$ is vertex-transitive and such that $T_{i}, 1 \leqslant i<k$, is a star rooted at its center $x_{i}$. If $G$ has a pruning and no *-fixed pendant vertex, the star roots induce a reducible partition of $V\left(G^{\sharp}\right)$ under $A\left(G^{\#}\right)$ by the rule: $X_{j}=\{x: x$ is the root of a $j$-star $\}$. Conversely, any reducible partition of $V\left(G^{\sharp}\right)$ leads to a graph $G$ with a pruning which induces the given reducible partition. Because of this equivalence $t\left(A\left(G^{\sharp}\right), V\left(G^{\sharp}\right)\right)$ is termed the tree growth number of $G^{\sharp}$.

\section{Lemmas on permutation groups.}

LEMMA 1. Let $A$ be a group acting on $a$ set $X$ with $a$ reducible partition $X=\cup_{i=0}^{s} X_{i}$. Let $Z \subseteq X_{i}$, for some $i>1$, and let $H$ be a subgroup such that either (1) $H \subset A_{\left(X_{i}^{\prime}\right)}$ where $Z \subseteq X_{i}^{\prime} \subseteq X_{i}$ and $(z) \alpha_{z^{\prime}} \in X_{i}^{\prime}$ for all distinct $z, z^{\prime} \in Z$, or (2) $H \subset A_{\left(X_{i-1}^{\prime}\right)}$ where $I(Z) \subseteq X_{i-1}^{\prime} \subseteq X_{i-1}$. If $K=\left\langle H,\left\{\alpha_{z}: z \in Z\right\}\right\rangle$ then $\left.o(K)\right\rangle$ $(|Z|+1) o(H)$. 
Proof. Note that if $o(H)<1$ or $|Z| \leqslant 1$ the result is clear. Let $\psi, \varphi \in H$ and $z, z^{\prime} \in Z$, where $\psi \neq \varphi$ and $z \neq z^{\prime}$. Then $\alpha_{z} \psi \neq \alpha_{z} \varphi, \alpha_{z} \psi \neq \varphi$, and $\alpha_{z} \psi \neq \alpha_{z^{\prime}} \psi$. Suppose $\alpha_{z} \psi=\alpha_{z^{\prime}} \varphi$. Suppose $H \subset A_{\left(X_{i}^{\prime}\right)}$. Then $(z) \alpha_{z} \psi \notin X_{i}^{\prime}$, but $(z) \alpha_{z^{\prime}} \varphi \in X_{i}^{\prime}$. Suppose $H \subset A_{\left(X_{i-1}^{\prime}\right)}$. Then $(z) \alpha_{z} \psi \in X_{i-1}^{\prime}$, but $(z) \alpha_{z^{\prime}} \varphi \notin X_{i-1}^{\prime}$. Hence $o(K)>$ $(|Z|+1) o(H)$.

LEMMA 2. Let $\left\{n_{i}\right\}_{i=1}^{s}$ be a sequence of positive integers with $s>2$. If $\sum_{i=1}^{s} n_{i}=N$ then $\Pi_{i=1}^{s}\left(n_{i}+1\right) \geqslant 2 N$.

Proof. By induction on $s$.

LEMMA 3. Let $A$ be a group acting on $a$ set $X$ with a reducible partition $X=\cup_{j=0}^{s} X_{j}$. Suppose $Z \subseteq X_{j}$ for $1<j<t$ and let $H=\left\langle\alpha_{z}: z \in Z\right\rangle$ and for every reducing orbit $O$ of $H O \cap X_{j} \subset Z$. Then either $O(H)=2^{a}=|Z|+1$ for some positive integer a or $O(H)>2|Z|$.

Proof. Let $H$ have $k \geqslant 2$ orbits $O_{1}, \ldots, O_{k}$ such that $Z_{i}=O_{i} \cap Z \neq \varnothing$. Let $H_{1}=\left\langle\alpha_{z}: z \in Z_{1}\right\rangle$. Clearly, $\left.o\left(H_{1}\right)\right\rangle\left|Z_{1}\right|+1$. Since $H_{1} \subset H_{\left(Z_{2}\right)}$ by Lemma 1 (L1), $o\left(H_{2}\right)>\left(\left|Z_{2}\right|+1\right) o\left(H_{1}\right)$ where $H_{2}=\left\langle\alpha_{z}: z \in Z_{1} \cup Z_{2}\right\rangle$. By the repeated application of (L1), $o(H)>\Pi_{i=1}^{k}\left(\left|Z_{i}\right|+1\right)$. By (L2), $o(H)>2|Z|$.

Let $O$ be the only orbit of $H$ such that $Z \cap O \neq \varnothing$. Let $\mid$ denote divides. Now $|O| \mid o(H)$. If $|O|<o(H)$ then $o(H) \geqslant 2|O|>2|Z|$. If $|O|=o(H)$ then $H$ is regular on $O$. Suppose $|O|=|Z|+1$. Then $(z) \alpha_{z}^{2}=z$ and by regularity $\alpha_{z}$ has order 2, thus by Cayley's Theorem $o(H)=2^{a}$. Suppose $|O|>|Z|+1$. Let $X_{j-1}^{\prime}=$ $O \cap X_{j-1}$. A reducing map sends only one element of $X_{j-1}^{\prime}$ into $Z$. The remaining images of $X_{j-1}^{\prime}$ lie in $X_{j-1}^{\prime}$. Since $H$ is regular on $O, \mid\left\{(p, q) \mid p \in X_{j-1}^{\prime}\right.$ and $q=(p) \psi \in X_{j-1}^{\prime}$, where $\left.\psi \in H\right\}\left.|=| X_{j-1}^{\prime}\right|^{2}$. Each $\alpha_{z}$ induces $\left|X_{j-1}^{\prime}\right|-1$ of these pairs. The identity map induces $\left|X_{j-1}^{\prime}\right|$ pairs. Hence $\left(\left|X_{j-1}^{\prime}\right|-1\right)|Z|<\left|X_{j-1}^{\prime}\right|^{2}-$ $\left|X_{j-1}^{\prime}\right|$ so that $|Z| \leqslant\left|X_{j-1}^{\prime}\right|$. Hence $o(H)=|O|>\left|X_{j-1}^{\prime}\right|+|Z|>2|Z|$.

COROLlaRY 3.1. Let $A$ be a group acting on a set $X$. Let $s>2$ and let $\cup_{i=0}^{s} X_{i}$ be a reducible partition of $X$. Let $H \subseteq A$ and suppose $H$ has an orbit $O$ such that $O \cap X_{j} \neq \varnothing$ and $O \cap X_{j+1} \neq \varnothing$, for some $1<j<s$. Suppose $\left\langle\alpha_{z}: z \in O \cap\left(X_{j}\right.\right.$ $\left.\cup X_{j+1}\right)>\subset H$. Then $H$ is not regular on $O$.

Proof. Let $W=O-\left(X_{j} \cup X_{j+1}\right)$. Suppose $H$ is regular on $O$. Since $H$ is regular, each point $x \in O$ is the image of $y \in O$ by a single map of $H$. Reducing $X_{j} \cap O$ and $X_{j+1} \cap O$ requires $\left|\left(X_{j} \cup X_{j+1}\right) \cap O\right|$ distinct nonidentity maps of $H$ by definition. Let $v \in X_{j+1}$. No reducing map $\alpha_{x}$ for $x \in O \cap\left(X_{j} \cup X_{j+1}\right)$ is such that $(w) \alpha_{x}=v$ where $w \in W$. Hence there are $|W|$ such maps in $H$. However, the total number of nonidentity maps in $H$ is only $|O|-1$, a contradiction. Hence $H$ is not regular on $O$.

Corollary 3.2. Let $A$ be a group acting on a set $X$. Let $X=\cup_{i=0}^{s} X_{i}$ be $a$ reducible partition. Let $H=\left\langle\alpha_{z}: z \in X_{k}\right\rangle$ for some $\left.k\right\rangle 0$.

(1) Suppose $H$ has a single reducing orbit $O$. Then

(a) $o(H)=\left|X_{k}\right|+1$ when $\left|X_{k}\right|=2^{a}-1$ for $a>1$.

(b) $o(H)=2\left|X_{k}\right|$ when $\left|O \cap X_{k-1}\right|=\left|X_{k}\right|$, and 
(c) $o(H) \geqslant 2\left(\left|X_{k}\right|+1\right)$ otherwise.

(2) Suppose $H$ has exactly two reducing orbits $O_{1}$ and $O_{2}$. Then

(a) $o(H)>2\left|X_{k}\right|$, or

(b) $o(H)=2\left|X_{k}\right|,\left|O_{1}\right|=2$, and $\left|O_{2}\right|=\left|X_{k}\right|=2^{b}$ for $b>1$.

(3) Suppose $H$ acts on $l \geqslant 3$ reducing orbits. Then

(a) $o(H) \geqslant 3\left|X_{k}\right|$ if $\left|X_{k}\right|>p$, and

(b) $o(H) \geqslant 2^{p}$ if $\left|X_{k}\right|=p$.

Proof. Suppose $H$ has a single reducing orbit $O$. If $H$ does not act regularly on $O$ then $o\left(H_{x}\right) \geqslant 2$ for $x \in O$. Since $O$ contains a point of $X_{k-1}, o(H)>$ $2\left(\left|X_{k}\right|+1\right)$. Suppose $H$ acts regularly on $O$. By (L3), $o(H)=\left|X_{k}\right|+1$ only if $\left|X_{k}\right|=2^{a}-1$, for $a>1$. By (L3), $\left|X_{k-1} \cap O\right|>\left|X_{k}\right|$ if $\left|X_{k-1} \cap O\right|>2$. Hence if $o(H)=2\left|X_{k}\right|$ then $\left|X_{k-1} \cap O\right|=\left|X_{k}\right|$. Suppose $H$ acts on two reducing orbits $O_{1}$ and $O_{2}$. Let $\left|O_{1} \cap X_{k}\right|=b$ and $\left|O_{2} \cap X_{k}\right|=a$ and suppose $a>b$. By (L1), $o(H)>(a+1)(b+1)>a b+a+b+1$. If $b>2$ then $o(H)>2\left|X_{k}\right|$. If $b=1$ then $o(H) \geqslant 2(a+b)$. Hence, if $o\left(H_{1}\right)=2\left|X_{k}\right|$ then $\left|O_{1} \cap X_{k}\right|=1$. The unique point of $O_{1} \cap X_{k}$ is fixed by $H^{\prime}=\left\langle\alpha_{z}: z \in O_{2} \cap X_{k}\right\rangle$. Since $o(H)<o\left(H^{\prime}\right)\left|O_{1}\right|$ it follows that $\left|O_{1}\right|=2$. Since $o\left(H^{\prime}\right)=\left|O_{2} \cap X_{k}\right|+1$ it follows by (L3) that $\left|X_{k}\right|=$ $2^{c}$ for $c \geqslant 1$.

Suppose $H$ acts on $p>3$ orbits $O_{1}, \ldots, O_{p}$. By $(\mathrm{L} 1), o(H)>\Pi_{j=1}^{p}\left(a_{j}+1\right)$ where $a_{j}=\left|X_{k} \cap O_{j}\right|$ for $1 \leqslant j \leqslant p$. Without loss of generality, $\left\{a_{j}\right\}_{j=1}^{p}$ is ordered so that $a_{j} \geqslant a_{j-1}$ for $2<j<p$. Suppose $a_{2} \geqslant 2$. Then

$$
\begin{aligned}
\left(a_{p}+1\right) & \left(a_{p-1}+1\right) \cdots\left(a_{1}+1\right) \\
& \geqslant a_{p}+\cdots+a_{1}+a_{p}\left(a_{p-1}+\cdots+a_{1}\right)+a_{p}\left(a_{p-1} a_{p-2}+\cdots+a_{2} a_{1}\right) \\
& \geqslant 3\left|X_{k}\right| \quad \text { if } p \geqslant 4 .
\end{aligned}
$$

If $p=3$ and $a_{2} \geqslant 2$ then

$$
\begin{gathered}
\left(a_{3}+1\right)\left(a_{2}+1\right)\left(a_{1}+1\right)>a_{2} a_{1}+a_{1}+a_{2}+1+a_{3} a_{1}+a_{3} a_{2}+a_{3} \\
\geqslant\left(a_{1}+a_{2}+a_{3}\right)+\left(a_{2} a_{1}+a_{3} a_{2}+a_{3} a_{1} a_{2}\right)>3\left|X_{k}\right| .
\end{gathered}
$$

If $a_{2}=1$ then $\left(a_{3}+1\right)\left(a_{2}+1\right)\left(a_{1}+1\right)=\left(a_{3}+1\right) 4>3 a_{3}+a_{3}+4>3\left|X_{k}\right|$.

If $a_{p}=1$ then $\left|X_{k}\right|=p$ and $o\left(H_{1}\right) \geqslant 2^{p}$ by (L1).

LEMMA 4. Let $A$ be a group acting faithfully and transitively on a set $X$. Suppose $X=X_{0} \cup X_{1} \cup X_{2}$ is a reducible partition. Then neither $A_{\left(X_{2}\right)}$ nor $A_{\left(X_{0}\right)}$ contains a nontrivial normal subgroup of $A$.

Proof. Let $N$ be a nontrivial normal subgroup of $A$. The orbits of $N$ are blocks of $A$. Since $A$ is faithful these orbits are not singletons. Let $x_{2} \in X_{2}$ and let $\left(x_{2}\right) \alpha_{x_{2}}=x_{1}$. Suppose $N \subset A_{\left(x_{2}\right)}$ so there exists $\psi \in N$ with a cycle $\left(x_{2} x_{2}^{\prime} \cdots\right)$, $x_{2}^{\prime} \in X_{2}$. Then $\left(x_{2}^{\prime}\right) \alpha_{x_{2}} \in X_{2}$ by definition, and $\alpha_{x_{2}}^{-1} \psi \alpha_{x_{2}}$ has a cycle $\left(x_{1}\left(x_{2}^{\prime}\right) \alpha_{x_{2}} \cdots\right)$. However, $\alpha_{x_{2}}^{-1} \psi \alpha_{x_{2}} \in N$ while $\alpha_{x_{2}}^{-1} \psi \alpha_{x_{2}} \notin A_{\left(x_{2}\right)}$, a contradiction. Suppose $N \subset A_{\left(x_{0}\right)}$. Let $x_{1} \in X_{1}$ and $\left(x_{1}\right) \alpha_{x_{1}}=x_{0}$. There exists a $\psi \in N$ with a cycle $\left(x_{1} x_{1}^{\prime} \cdots\right)$ with $x_{1}^{\prime} \in X_{1} \cup X_{2}$. Then $\left(x_{1}^{\prime}\right) \alpha_{x_{1}} \in X_{1} \cup X_{2}$ and $\alpha_{x_{1}}^{-1} \psi \alpha_{x_{1}}$ has a cycle $\left(x_{0}\left(x_{1}^{\prime}\right) \alpha_{x_{1}} \cdots\right)$. However, $\alpha_{x_{1}}^{-1} \psi \alpha_{x_{1}} \in N$ and $\alpha_{x_{1}}^{-1} \psi \alpha_{x_{1}} \notin A_{\left(x_{0}\right)}$, a contradiction. 
REMARK. Actually, the hypothesis that $X_{0} \cup X_{1} \cup X_{2}$ is reducible can be weakened to the existence of some reducing maps $\alpha_{x_{2}}$ and $\alpha_{x_{1}}$.

Corollary 4.1. Let $H=\left\langle\alpha_{z}: z \in X_{2}\right\rangle$ and let $K=\left\langle\alpha_{z}: z \in X_{1}\right\rangle$. Then $o(A) \mid[A: K] !$ and $o(A) \mid[A: H]$ !.

Proof. By (L4), $K$ and $H$ cannot contain normal subgroups of $A$. However, by the core theorem of algebra (Herstein, Topics in algebra, Blaisdell, Waltham, Mass., 1964, p. 62), $o(A) \mid[A: K] !$ and $o(A) \mid[A: H] !$.

Corollary 4.2. Let $A$ act on a set $X$. Let $X=\cup_{i=0}^{i} X_{i}$ be a reducible partition. Suppose $2<j \leqslant t$. Let $H_{1}=\left\langle\alpha_{z}: z \in X_{j}\right\rangle$ and $H_{2}=\left\langle\alpha_{z}: z \in X_{j} \cup X_{j-1}\right\rangle$. Suppose $O_{1}, \ldots, O_{k}$ are the reducing orbits of $H_{2}$ and $N_{2}$ is the pointwise stabilizer of $\cup_{v=1}^{k} O_{v}$. Let $\bar{H}_{2}=H_{2} / N_{2}$. Let $H_{1}^{\prime}=\left\langle H_{1}, N_{2}\right\rangle$ and $\bar{H}_{1}^{\prime}=H_{1}^{\prime} / N_{2}$. Then $\bar{H}_{1}^{\prime}$ contains no normal nontrivial subgroup of $\bar{H}_{2}$.

Proof. Suppose $N$ is a normal nontrivial subgroup of $\bar{H}_{2}$ contained in $\bar{H}_{1}^{\prime}$. Then $N$ acts nontrivially on an orbit $O_{s}$ for $1 \leqslant s \leqslant k$. Also $O_{s} \cap X_{j-1} \neq \varnothing$, since $O_{s}$ is a reducing orbit. Let $x_{j-1} \in O_{s} \cap X_{j-1}$. Since the orbits of $N$ are blocks of $\bar{H}_{2}$ there exists a $g \in N$ containing a nontrivial cycle $\left(x_{j-1} x_{j-1}^{\prime} \cdots\right)$ where $x_{j-1}^{\prime} \in X_{j-1} \cup$ $X_{j}$. Then $\left(x_{j-1}\right) \alpha_{x_{j-1}} \in X_{j-2}$ and $\left(x_{j-1}^{\prime}\right) \alpha_{x_{j-1}} \in X_{j-1} \cup X_{j}$ by definition. Since $\alpha_{x_{j-1}}^{-1}$ $\cdot g \alpha_{x_{j-1}}$ contains the cycle $\left(\left(x_{j-1}\right) \alpha_{x_{j-1}}\left(x_{j-1}^{\prime}\right) \alpha_{x_{j-1}} \cdots\right)$ a contradiction follows from $N \subset \bar{H}_{1}^{\prime} \subset \bar{H}_{2\left(X_{j-2}\right)}$. Hence, $H_{1}^{\prime}$ contains no normal subgroup of $\bar{H}_{2}$. As in (L4C1) this means $o\left(\bar{H}_{2}\right) \mid\left[\bar{H}_{2}: \bar{H}_{1}^{\prime}\right]$ !.

Lemma 5. Let $A$ be a group acting on a set $X$. Let $X=\cup_{i=0}^{s} X_{i}$ be a reducible partition. Suppose $1 \leqslant j \leqslant s$ is such that $\left|X_{j}\right| \geqslant 2$. Suppose $H$ is a subgroup of $A$ that stabilizes $X_{j}$. Let $K=\left\langle H,\left\{\alpha_{z}: z \in X_{j}\right\}\right\rangle$. Then there exists $x \in X_{j}$ such that $o\left(K_{x}\right) \geqslant 2 o(H) /\left|X_{j}\right|$.

Proof. Suppose $X_{j}$ is not an orbit of $H$. There exists an orbit $O$ of $H$ such that $O \subseteq X_{j}$ and $|O| \leqslant\left|X_{j}\right| / 2$. Hence $o\left(K_{x}\right)>o\left(H_{x}\right)>2 o(H) /\left|X_{j}\right|$ for any $x \in O$. Suppose $X_{j}$ is an orbit of $H$. Let $x, x^{\prime}$ be distinct elements of $X_{j}$. Since $X_{j}$ is an orbit of $H$ there are $o(H) /\left|X_{j}\right|$ maps of $H$ sending $x^{\prime}$ to $\left(x^{\prime}\right) \alpha_{x}$. Let $\psi$ be such a map. Then $\left(x^{\prime}\right) \alpha_{x} \psi^{-1}=x^{\prime}$ and also $\alpha_{x} \psi^{-1} \notin H_{x^{\prime}}$. Hence $o\left(K_{x^{\prime}}\right)>2 o\left(H_{x^{\prime}}\right)>$. $2 o(H) /\left|X_{j}\right|$.

Let $A$ act on a set $X$. Let $X=\cup_{i=0}^{t} X_{i}$ be a reducible partition. Suppose $1<j \leqslant t$ and $t \geqslant 2$. Let

$$
H_{l}=\left\langle\alpha_{z}: z \in X_{j} \cup \cdots \cup X_{j-(l-1)}\right\rangle \quad \text { if } 1<l<j
$$

and

$$
H_{l}=\left\langle\alpha_{z}: z \in X_{1} \cup \cdots \cup X_{l}\right\rangle \text { if } j<l<t .
$$

Let $O_{t}$ be a reducing orbit of $H_{t}$ that intersects $X_{t}$. Let $O_{t-1}$ be a reducing orbit of $H_{t-1}$ contained within $O_{t}$ which intersects $X_{t-1}$ if $j<t$ and intersects $X_{t}$ if $j=t$. Let $O_{t-i} \subseteq O_{t-(i-1)}$ be a reducing orbit of $H_{t-i}$ for $2<i<t-1$, such that $O_{t-i}$ intersects $X_{t-i}$ if $j \leqslant t-i$ and intersects $X_{j}$ if $t-i<j$. For $2<l<t$ define $N_{l}$ to 
be the subgroup of $H_{l}$ that stabilizes $O_{l}$ pointwise. Let

$$
\begin{aligned}
& \bar{H}_{l}=H_{l} / N_{l} \quad \text { if } 1<l<t-1, \\
& H_{l}^{\prime}=\left\langle H_{l}, N_{l+1}\right\rangle \quad \text { if } 2<l<t, \text { and } \\
& \bar{H}_{l}^{\prime}=H_{l}^{\prime} / N_{l+1} \quad \text { if } 2<l<t .
\end{aligned}
$$

Lemma 6. Let $A$ act on a set $X$. Let $X=\cup_{i=0}^{i} X_{i}$ be a reducible partition. Suppose $1 \leqslant j \leqslant t$ and $t \geqslant 2$. For $2<l<t$, $u_{l}$ is defined to be the least positive integer such that $u_{l} ! \geqslant u_{l} o\left(\bar{H}_{l-1}^{\prime}\right)$ where $\bar{H}_{l-1}^{\prime}$ is defined in (2.2). Then for $l>i>2$, $o\left(H_{l}\right) \geqslant u_{l} \cdots u_{i} o\left(H_{i-1}\right)$ where $u_{l}>\cdots>u_{i}>i+1$ and where $H_{l}$ and $H_{i-1}$ are defined in (2.1). Moreover, $o\left(H_{2}\right)>o\left(\bar{H}_{2}\right) \geqslant 3 !\left|X_{j}\right|$.

Proof. First it is shown that $o\left(H_{l+1}\right) \geqslant u_{l+1} o\left(H_{l}\right)$ for $1<l<t-1$. By (L4 C1), $o\left(\bar{H}_{l+1}\right) \mid\left[\bar{H}_{l+1}: \bar{H}_{l}^{\prime}\right]$ !. Hence $\left[\bar{H}_{l+1}: \bar{H}_{l}^{\prime}\right] !>\left[\bar{H}_{l+1}: \bar{H}_{l}\right] o\left(\bar{H}_{l}^{\prime}\right)$. Hence $\left[\bar{H}_{l+1}: \bar{H}_{l}^{\prime}\right]>$ $u_{l+1}$. By the correspondence theorem $\left[\bar{H}_{l+1}: \bar{H}_{l}\right]=\left[H_{l+1}: H_{l}^{\prime}\right]>u_{l+1}$. Since $H_{l}^{\prime}>$ $H_{l},\left[H_{l+1}: H_{l}\right] \geqslant\left[H_{l+1}: H_{l}^{\prime}\right]$. Hence $o\left(H_{l+1}\right)>u_{l+1} o\left(H_{l}\right)$.

Next it is shown that $u_{l+1}>u_{l}$ for $2<l<t-1$. Suppose $u_{l+1}<u_{l}$. By definition $u_{l+1} ! \geqslant u_{l+1} o\left(\bar{H}_{l}^{\prime}\right)$. Hence $u_{l} ! \geqslant u_{l} o\left(\bar{H}_{l}^{\prime}\right)$ and $\left(u_{l}-1\right) ! \geqslant o\left(\bar{H}_{l}^{\prime}\right)$. Since $\left[\bar{H}_{l}: \bar{H}_{l-1}^{\prime}\right] \geqslant u_{l}, o\left(\bar{H}_{l}\right) \geqslant u_{l} o\left(\bar{H}_{l-1}^{\prime}\right)$.

$$
o\left(\bar{H}_{l}^{\prime}\right)=\frac{o\left(H_{l}\right) o\left(N_{l+1}\right)}{o\left(H_{l} \cap N_{l+1}\right) o\left(N_{l+1}\right)}=\frac{o\left(H_{l}\right)}{o\left(H_{l} \cap N_{l+1}\right)}
$$

and

$$
o\left(\bar{H}_{l}\right)=\frac{o\left(H_{l}\right)}{o\left(H_{l} \cap N_{l}\right)} .
$$

Now $H_{l} \cap N_{l} \supset H_{l} \cap N_{l+1}$ since if $g \in H_{l} \cap N_{l+1}$ it fixes all points $O_{l}$ and since it is in $H_{l}$ it is in $N_{l}$. Hence $o\left(\bar{H}_{l}^{\prime}\right)>o\left(\bar{H}_{l}\right)$. But then, $\left(u_{l-1}\right) !>o\left(\bar{H}_{l}\right)$, whence $\left(u_{l-1}\right) ! \geqslant u_{l} o\left(\bar{H}_{l-1}^{\prime}\right)$, a contradiction of the choice of $u_{l}$.

Finally, it is shown that $o\left(H_{2}\right)>6\left|X_{j}\right|$. Note since $o\left(\bar{H}_{1}^{\prime}\right)>2, u_{2}>3$. If $o\left(H_{1}\right)>$ $2\left|X_{j}\right|$ then, since $u_{2}>3, o\left(H_{2}\right)>3 !\left|X_{j}\right|$. Otherwise, by (L3), $o\left(H_{1}\right)=2^{a}=\left|X_{j}\right|+1$ for some $a \geqslant 1$.

Suppose $a=1$. In this case $\left|X_{j}\right|=1$. Since $o\left(H_{1}\right)=2$ and $u_{2}>3, o\left(H_{2}\right)>6\left|X_{j}\right|$.

Suppose $a>4$. In this case, $\left|X_{j}\right| \geqslant 15$. Since $H_{1}$ is regular $H_{1} \cap N_{2}=\langle e\rangle$ so $o\left(\bar{H}_{1}^{\prime}\right)=o\left(H_{1}\right) / o\left(H_{1} \cap N_{2}\right)=o\left(H_{1}\right)$. Hence $16 \mid o\left(\bar{H}_{1}^{\prime}\right)$ and hence $\left[\bar{H}_{2}: \bar{H}_{1}^{\prime}\right]>7$ by (L4 C1). Thus $o\left(H_{2}\right) \geqslant 6\left|X_{j}\right|$.

Suppose $a=2$. In this case, $\left|X_{j}\right|=3$ and $o\left(H_{1}\right)=4$. Since $H_{1}$ is regular $o\left(H_{1}\right)=$ $o\left(\bar{H}_{1}^{\prime}\right)=4$. By $(\mathrm{L} 4 \mathrm{Cl}), o\left(\bar{H}_{2}\right) \mid\left[\bar{H}_{2}: \bar{H}_{1}^{\prime}\right]$ !. Hence $\left[\bar{H}_{2}: \bar{H}_{1}^{\prime}\right]>5$ since $4^{2} \nmid 4$ !. Thus $o\left(H_{2}\right)>5 \cdot 4>6\left|X_{j}\right|$.

Suppose $a=3$. In this case, $\left|X_{j}\right|=7$ and $o\left(H_{1}\right)=8$. Since $H_{1}$ is regular $o\left(\bar{H}_{1}^{\prime}\right)=$ 8. If $\left[\bar{H}_{2}: \bar{H}_{1}^{\prime}\right]>6$ the result follows. Hence $\left[\bar{H}_{2}: \bar{H}_{1}^{\prime}\right]=5$. Hence $o\left(\bar{H}_{2}\right)=40$.

Suppose $j=1$. Then $\left|X_{2}\right|<4$ for otherwise using (L1), $o\left(H_{2}\right)>\left(\left|X_{2}\right|+1\right) o\left(H_{1}\right)$ $>6 \cdot 8>3 !\left|X_{1}\right|$. Suppose $\left|X_{2}\right|=3$ or 4 . Since $\left|O_{2}\right|$ must divide 40 and $\left|O_{2}\right|>11$, $\left|O_{2}\right|=20$ or 40 . Since $X_{2}$ is stabilized setwise by $\bar{H}_{1}^{\prime}$, by (L5), $o\left(\left(\bar{H}_{2}\right)_{x_{2}}\right)>4$, a contradiction of $\left|O_{2}\right|=20$ or 40 by (P7.4). If $\left|X_{2}\right|=2$ or 1 then by (L5), $o\left(\left(\bar{H}_{2}\right)_{x_{2}}\right)$ $>8$, a contradiction to $\left|O_{2}\right|>10$. 
Suppose $j>1$. Let $x_{j-1}$ be the point of $X_{j-1}$ in the reducing orbit of $\bar{H}_{1}^{\prime}$. Suppose $\left|X_{j-1}\right| \neq 1$. Note $X_{j-1}-\left\{x_{j-1}\right\}$ is stabilized by $\bar{H}_{1}^{\prime}$ and $\alpha_{x_{j-1}}$. If $\bar{H}$ is generated by $\bar{H}_{1}^{\prime}$ and $\alpha_{x_{j-1}}$, then $o(\bar{H}) \geqslant 40$ by (L4 C1), a contradiction to $o(\bar{H})<o\left(\bar{H}_{2}\right)=40$. Let $\left|X_{j-1}\right|=1$. Then $\left|O_{2}\right| \mid 40$. Since $\left|X_{j} \cup X_{j-1}\right|=8,\left|X_{j-2} \cap O_{2}\right|=2$, 12, or 32 . Suppose $\left|O_{2} \cap X_{j-2}\right|=2$. Since $3 \nmid o\left(H_{2}\right), \alpha_{x_{j-1}}$ has cycles $\left(x_{j-2}\right)\left(x_{j-2}^{\prime} x_{j-1}\right)$. Since $X_{j-2} \cap O_{2}$ is stabilized by $\bar{H}_{1}^{\prime}, o\left(\left(\bar{H}_{1}^{\prime}\right)_{x_{j-2}}\right) \geqslant 4$. Hence $o\left(\left(\bar{H}_{2}\right)_{x_{j-2}}\right)>8$, a contradiction to $o\left(\left(\bar{H}_{2}\right)_{x_{j-2}}\right)=o\left(\bar{H}_{2}\right) /\left|O_{2}\right|=4$. Let $\left|X_{j-2} \cap O_{2}\right|=12$. Since $C_{5}$ is a normal subgroup of $\bar{H}_{2}$ the orbits of $C_{5}$ are a complete block system of $\bar{H}_{2}$. No point of $X_{j}$ in a block with $x_{j-1}$ can be reduced since the reducing maps of $X_{j}$ do not have order 5 . Hence the block containing $x_{j-1}$ contains 4 points of $X_{j-2}$. However, if $\left(x_{j}\right) \alpha_{x_{j}}=x_{j-1}, x_{j}$ is in a block containing 4 distinct points of $X_{j-2}$. Since this can be true for only two $x_{j}$, a contradiction follows. Let $\left|X_{j-2} \cap O_{2}\right|=32$. Then $\bar{H}_{2}$ is regular on $\mathrm{O}_{2}$ and hence by $(\mathrm{L} 3 \mathrm{C} 1)$ cannot reduce $\mathrm{O}_{2} \cap\left(X_{j} \cup X_{j-1}\right)$. Hence (L6) is proved.

Corollary 6.1. Let $A$ act on a set $X$. Let $X=\cup_{i=0}^{t} X_{i}$ be a reducible partition. Then $o(A) \geqslant(t+1)$ ! and $o(A)=(t+1)$ ! only if there is a reducing orbit $O$ of $A$ such that $|O|=t+1$ and $A^{0} \simeq S_{t+1}$.

Proof. Let $O$ be a reducing orbit of $A$ such that $\left|O \cap X_{t}\right|>1$. Let $H_{t}=\left\langle\alpha_{z}\right.$ : $z \in O \cap \cup_{i>1} X_{i}>$. If $t=1$ then $o(A) \geqslant 2$ and $o(A)=2$ implies $A^{0} \simeq C_{2}$ and the result is clear. If $t>1$ then by (L6) for every $j>1, o\left(H_{t}\right)>(t+1) !\left|X_{j}\right|$. Hence $o(A) \geqslant(t+1)$ ! and each $X_{j}$ is a singleton for $j \geqslant 1$ if $o(A)=(t+1)$ !. Suppose $o(A)=(t+1)$ !. Let $H_{t-1}=\left\langle\alpha_{z}: z \in O \cap \cup_{i=1}^{t-1} X_{i}\right\rangle$. Then $o\left(H_{t-1}\right)>t$ ! by (L6). But $H_{t-1}$ fixes $X_{t}$. Thus $o\left(A^{0}\right) \leqslant|O| t$ ! and hence $|O|=t+1$. Hence $A^{0} \cong S_{t+1}$.

COROLlaRY 6.2. Let $A$ act on a set $X$. Let $X=\cup_{i=0}^{t} X_{i}$ be a reducible partition. Let $H_{1}=\left\langle\alpha_{z}: z \in X_{j}\right\rangle$. Suppose either

$$
H_{2}=\left\langle\alpha_{z}: z \in X_{j} \cup X_{j+1}\right\rangle \text { and } H_{3}=\left\langle\alpha_{z}: z \in X_{j} \cup X_{j+1} \cup X_{j+2}\right\rangle
$$

and there is a reducing orbit $\mathrm{O}_{2}$ of $\mathrm{H}_{2}$ meeting $X_{j}$ in at least 2 points, or

$$
H_{2}=\left\langle\alpha_{z}: z \in X_{j-1} \cup X_{j}\right\rangle \text { and } H_{3}=\left\langle\alpha_{z}: z \in X_{j-2} \cup X_{j-1} \cup X_{j}\right\rangle
$$

and $\left|X_{j}\right|>2$.

Then $o\left(H_{3}\right) \geqslant o\left(\bar{H}_{3}\right) \geqslant 120$ where $\bar{H}_{3}$ is defined as in (2.2).

Proof. Let $H_{2}$ and $H_{3}$ be as in (2.4). Let $\bar{H}_{2}$ and $\bar{H}_{1}^{\prime}$ be as in (2.2) acting on the orbit $\mathrm{O}_{2}$. Since $\left|X_{j} \cap \mathrm{O}_{2}\right| \geqslant 2, o\left(\bar{H}_{1}^{\prime}\right) \geqslant 4$ by (L3). Suppose $o\left(\bar{H}_{1}\right)=4$. By (L4 Cl), $\left[\bar{H}_{2}: \bar{H}_{1}^{\prime}\right] \geqslant 5$. Also $o\left(\bar{H}_{1}^{\prime}\right) \neq 5$ since $C_{5}$ has only a single reducing map. Suppose $o\left(\bar{H}_{1}\right)=6$; then $\left[\bar{H}_{2}: \bar{H}_{1}^{\prime}\right] \geqslant 4$ by $(\mathrm{L} 4 \mathrm{Cl})$. Suppose $o\left(\bar{H}_{1}\right) \geqslant 7$; then $\left[\bar{H}_{2}: \bar{H}_{1}\right]>$ 5. Using the calculation of $(2.3), o\left(H_{2}^{\prime}\right) \geqslant o\left(\bar{H}_{2}\right)$. Suppose $o\left(\bar{H}_{2}^{\prime}\right)>24$; then by (L4 C1), $\left[\bar{H}_{3}: \bar{H}_{2}^{\prime}\right] \geqslant 5$ and $o\left(\bar{H}_{3}\right)>120$.

Let $\mathrm{H}_{3}$ and $\mathrm{H}_{2}$ be as in (2.5). If there is a reducing orbit of $\mathrm{H}_{2}$ meeting $\mathrm{X}_{j}$ in at least 2 points the argument follows as above. Suppose not. Let $O_{21}, \ldots, O_{2 k}$ be the reducing orbits of $H_{2}$. Let $N_{2}$ be the pointwise stabilizer of $\cup_{i=1}^{k} O_{2 i}$. Let $\bar{H}_{2}=H_{2} / N_{2}$. Let $H_{1}^{\prime}=\left\langle H, N_{2}\right\rangle$ and let $\bar{H}_{1}^{\prime}=H_{1}^{\prime} / N_{2}$. By $\left.(\mathrm{L} 3), o\left(\bar{H}_{1}^{\prime}\right)\right\rangle 4$. This case now follows to the point of bounding $o\left(\bar{H}_{2}\right)$ as above using (L4 C2) in place of (L4 C1). Let $O_{31}, \ldots, O_{3 k^{\prime}}$ be the reducing orbits of $H_{3}$. Let $N_{3}$ be the pointwise 
stabilizer of $\cup_{i=1}^{k^{\prime}} O_{3 i}$. Let $\bar{H}_{3}=H_{3} / N_{3}$. Let $H_{2}^{\prime}=\left\langle H_{2}, N_{3}\right\rangle$ and let $\bar{H}_{2}^{\prime}=$ $H_{2}^{\prime} / N_{3}$. The case concludes as above using ( $\left.\mathrm{L} 4 \mathrm{C} 2\right)$ in place of $(\mathrm{L} 4 \mathrm{Cl})$.

Let $A$ act on a set $X$. Let $X=\cup_{i=0}^{t} X_{i}$ be a reducible partition. For $1<l<t$ let

$$
H_{l}=\left\langle\alpha_{z}: z \in X_{t-(l-1)} \cup \cdots \cup X_{t}\right\rangle .
$$

For $2 \leqslant l \leqslant t$ let $O_{l 1}, \ldots, O_{l k_{l}}$ be the reducing orbits of $H_{l}$. Let $N_{l}$ be the pointwise stabilizer $\cup_{v=1}^{k_{l}} O_{l v}$. For $2<l \leqslant t$, let

$$
\begin{aligned}
& \bar{H}_{l}=H_{l} / N_{l} \text { and for } 1 \leqslant l<t-1 \text { let } \\
& H_{l}^{\prime}=\left\langle H_{l}, N_{l+1}\right\rangle \text { and let } \\
& \bar{H}_{l}^{\prime}=H_{l}^{\prime} / N_{l+1} .
\end{aligned}
$$

Corollary 6.3. Let $A$ act on a set $X$. Let $X=\cup_{i=0}^{t} X_{i}$ be a reducible partition. Suppose $2 \leqslant l \leqslant t$ and $H_{1}$ has at least two reducing orbits. For $2<l<t$, $u_{l}$ is defined to be the least positive integer such that $u_{l} ! \geqslant u_{l} o\left(\bar{H}_{l-1}^{\prime}\right)$ where $\bar{H}_{l-1}^{\prime}$ is defined in (2.7). Then for $2 \leqslant i \leqslant l, o\left(H_{l}\right) \geqslant u_{l} \cdots u_{i} o\left(H_{i-1}\right)$ where $u_{l}>\cdots>u_{i}>i+1$ and where $H_{l}$ and $H_{i-1}$ are defined in (2.6). Moreover $u_{2} !>u_{2} o\left(\bar{H}_{1}^{\prime}\right)$.

Proof. By (L4 C2), $\left[\bar{H}_{l}: \bar{H}_{l-1}^{\prime}\right] \geqslant u_{l}$ for $2<l<t$ where $\bar{H}_{l}$ and $\bar{H}_{l-1}^{\prime}$ are defined. By the correspondence theorem $\left[\bar{H}_{l}: H_{l-1}^{\prime}\right]>u_{l}$. Since $o\left(H_{l-1}^{\prime}\right)>o\left(H_{l-1}\right)$, $o\left(H_{l}\right) \geqslant u_{l} o\left(H_{l-1}\right)$. Using the calculation in (2.3), it can be shown as in (L6) that for $3<l \leqslant t, u_{l}>u_{l-1}$ or the defining property of $u_{l-1}$ is contradicted. Hence for $2<l \leqslant t$ and $l \geqslant i \geqslant 2, o\left(H_{l}\right) \geqslant u_{l} \cdots u_{i} o\left(H_{i-1}\right)$ and $u_{l}>\cdots>u_{2}>3$. Since $\bar{H}_{1}^{\prime}$ acts on several reducing orbits, $o\left(\bar{H}_{1}^{\prime}\right) \geqslant 2\left|X_{t}\right|$ by (L3). Hence $u_{2} !>u_{2} \cdot 2 \cdot\left|X_{t}\right|$.

COROllary 6.4. Let $A$ act on a set $X$. Let $X=\cup_{i=0}^{t} X_{i}$ be a reducible partition. Suppose $t \geqslant 3$ and $\left|X_{t}\right| \geqslant 2$. Let $H_{l}$ and $\bar{H}_{l}$ be defined as in (2.6) and (2.7) for $1 \leqslant l \leqslant t$. Then for $3<l \leqslant t, o\left(H_{l}\right) \geqslant o\left(\bar{H}_{l}\right)>(l+2)$ !.

Proof. By (L6 C2), $o\left(\bar{H}_{3}\right) \geqslant 5$ ! if $\left|X_{t}\right| \geqslant 3$. Suppose it has been shown for $3 \leqslant l \leqslant t-1$ that $o\left(\bar{H}_{l}\right) \geqslant(l+2)$ !. By the calculation of $(2.3), o\left(\bar{H}_{l}^{\prime}\right)>o\left(\bar{H}_{l}\right)$. By the definition of $u_{l+1}, u_{l+1} \geqslant l+3$ since $o\left(\bar{H}_{l}^{\prime}\right)>(l+2)$ !. Hence $o\left(H_{l+1}\right)>$ $o\left(\bar{H}_{l+1}\right) \geqslant(l+3) o\left(H_{l}\right) \geqslant(l+3) !$.

LeMMA 7. Let $A$ be a group acting on a set $X$. Let $\cup_{i=0}^{s} X_{i}$ be a reducible partition of $X$. Let $H \subseteq A$. Suppose $Y$ is an orbit of $H$ where $Y \subset X_{j}, j>1$. Suppose $Z \subseteq X_{j+1}$ such that $I(Z) \subset Y$. Let $K=\left\langle H_{1},\left\langle\alpha_{z}: z \in Z\right\rangle\right\rangle$. Suppose that if $O$ is the orbit of $K$ containing $Y$, then $O \cap X_{j}=Y$. Let $L=\left\langle K,\left\langle\alpha_{y}: y \in Y\right\rangle\right\rangle$. Suppose that if $O^{\prime}$ is the orbit of $L$ containing $Y$ then $O^{\prime} \cap X_{j}=Y$. Let

$$
m=\min \{|Y|-1,|Z|\} \text {. }
$$

It is the case that $o\left(K_{y}\right) \geqslant(m+1) o\left(H_{y}\right)$, for $y \in Y$. Moreover, $o\left(L_{y}\right)>|Y| o\left(K_{y}\right)$.

Proof. The following propositions are used in the proof.

Proposition 7.1. Let $A$ be a group acting on a set $X$. Let $X=\cup_{i=0}^{s} X_{i}$ be a reducible partition. Suppose that $H \subseteq A$ and that $O$ is an orbit of $H$. Let $x \in O$ and let $(x) \psi_{x} \notin O$. Then if $h \in H, h \psi_{x}^{-1} \notin H$. 
Proof. Since $x \in O$ and $(x) \psi_{x} \notin O, \psi_{x} \notin H$. Hence $h \psi_{x}^{-1} \notin H$.

Proposition 7.2. Let $A$ be a group acting on a set $X$. Let $X=\cup_{i=0}^{s} X_{i}$ be $a$ reducible partition. Suppose that $H \subseteq A$ and that $O$ is an orbit of $H$. Suppose $\left\{z_{r}\right.$ : $1 \leqslant r \leqslant k\} \subset O$ is such that $\left(z_{r}\right) \alpha_{z_{r}} \notin O$ and $\left(z_{s}\right) \alpha_{z_{r}} \in O$ if $r \neq s$ for $1<r<k$ and $1<s \leqslant k$. If $h \in H, \alpha_{z_{s}}$ and $\alpha_{z_{r}}$ are such that $r \neq s$, then $h \alpha_{z_{s}}^{-1} \notin H$.

Proof. Since $\left(z_{s}\right) \alpha_{z_{s}} \in O$ and $O$ is an orbit of $H,\left(z_{s}\right) \alpha_{z_{s}} h^{-1} \notin O$. Now $\left((z) \alpha_{z_{s}} h^{-1}\right) h \alpha_{z_{s}} \alpha_{z_{r}}=\left(z_{s}\right) \alpha_{z_{r}} \in O$ by assumption. Since $O$ is an orbit of $H, h \alpha_{z_{s}}^{-1} \alpha_{z_{r}} \notin$ $H$.

Proposition 7.3. Let $A$ be a group acting on a set $X$. Let $X=\cup_{i=0}^{s} X_{i}$ be $a$ reducible partition. Let $H \subseteq A$. Let $Y$ be part of an orbit of $H$. Suppose $\alpha \notin H$ and (y) $\alpha \in Y$. Then there exists $h \in H$ such that $h \alpha^{-1} \in K_{y}$.

Proof. Since $Y$ is part of an orbit of $H$ there exists an $h$ such that $(y) h=(y) \alpha_{x}$. Hence $h \alpha_{x}^{-1} \in K_{y}$.

Proposition 7.4. Let $A$ be a group acting on a set $X$. Suppose $H \subseteq A$ and $O$ is an orbit of $H$. Then for every $x \in O, o\left(H_{x}\right)=o(H) /|O|$.

Proof. This is a basic fact of permutation group theory.

The main argument of the proof begins.

Since $|Z| \geqslant m$ there exists a set of distinct points $\left\{z_{1}, \ldots, z_{m}\right\}$ with $\left\{\alpha_{z_{1}}, \ldots, \alpha_{z_{m}}\right\}$ the set of corresponding reducing maps. Since $O \cap X_{j}=Y$, $\left(Y-\left(z_{k}\right) \alpha_{z_{k}}^{-1}\right) \alpha_{z_{k}} \subset Y$, for any $1 \leqslant k \leqslant m$. Hence at most $m$ distinct points of $Y$ are mapped outside $Y$ by one or more of the $\alpha_{z_{i}}, 1<i \leqslant m$. Hence since $|Y|-1>$ $m$, there exists a $y \in Y$ such that $(y) \alpha_{z_{i}} \in Y$, for $1<i<m$. By (P7.3) there exists an $h_{i} \in H$ for $1 \leqslant i<k$ such that $h_{i} \alpha_{z_{i}}^{-1} \in K_{y}$. By (P7.1), $h_{i} \alpha_{z_{i}}^{-1} \notin H_{y}$, for $1<i<$ $k$. Let $h$ and $h^{\prime}$ be two elements of $H_{y}$. If $h h_{i} \alpha_{z_{i}}^{-1}=h^{\prime} h_{k} \alpha_{z_{k}}^{-1}, i \neq k$, then $h h_{i}=$ $h^{\prime} h_{k} \alpha_{z_{k}}^{-1} \alpha_{z_{i}}$. By (P7.2), $h^{\prime} h \alpha_{z_{k}}^{-1} \notin H$, a contradiction. Thus for every map of $H_{y}$ there are $m+1$ maps of $K_{y}$, namely $h, h \alpha_{z_{1}}^{-1}, \ldots, h \alpha_{z_{m}}^{-1}$. The maps arising from different elements of $H_{y}$ are clearly distinct. Hence $o\left(K_{y}\right)>(m+1) o\left(H_{y}\right)$.

If $|Y|=1$, then $o\left(L_{y}\right) \geqslant o\left(K_{y}\right)$ since $L \supset K$. Suppose $|Y|>1$. Fix $y^{-} \in Y$. Let $y \in Y-y^{-}$. By (P7.3) there exists $k_{y} \in K$ such that $k_{y} \alpha_{y}^{-1} \in L_{y^{-}}$. By (P7.1) $k_{y} \alpha_{y}^{-1} \notin K_{y^{-}}$. There are $|Y|-1$ such maps. Suppose $k$ and $k^{\prime}$ are elements of $K_{y}$. Suppose $k k_{y^{\prime}} \alpha_{z_{y^{\prime}}}^{-1}=k^{\prime} k_{y} \alpha_{z_{y}}^{-1}$ where $y$ and $y^{\prime} \in Y-\left\{y^{-}\right\}$. Then $k k_{y^{\prime}}=k^{\prime} k_{y} \alpha_{z_{y}}^{-1} \alpha_{z_{y^{\prime}}}$, a contradiction of (P7.2). Hence for every map of $K_{y}$ there are $|Y|$ maps of $L_{y}$. Hence $o\left(L_{y}\right) \geqslant|Y| o\left(K_{y}\right)$.

Corollary 7.1. Let $A$ be a group acting on a set $X$. Let $X=\cup_{i=0}^{s} X_{i}$ be $a$ reducible partition of $X$. Let $H \subseteq A$. Suppose $Y=O \cap X_{j}$, for some $j>1$ and for some orbit $O$ of $H$. Suppose $Z$ is contained within an orbit $O_{Z}$ of $H$ distinct from $O$ and disjoint from $X_{0}$. Suppose for all distinct $z, z^{\prime} \in Z$ that $(z) \alpha_{z^{\prime}} \in Z$. Suppose $I(Z) \cap O_{Z}=\varnothing$. Let $K=\left\langle H,\left\langle\alpha_{z}: z \in Z\right\rangle\right\rangle$. Let $O^{\prime}$ be the orbit of $K$ containing $O$. Suppose $O^{\prime} \cap X_{1}=Y$. Suppose $\left\{(z) \alpha_{z}^{-1}: z \in Z\right\} \cap Y=\varnothing$. Then $o\left(K_{y}\right) \geqslant$ $(|Z|+1) o\left(H_{y}\right)$. 
Proof. Let $y \in Y$. Let $z \in Z$. Suppose $(y) \alpha_{z} \notin Y$. Then since $O^{\prime} \cap X_{j}=Y$, $(y) \alpha_{z}=z$. However, this contradicts the hypothesis that $\left\{(z) \alpha_{z}^{-1}: z \in Z\right\} \cap Y=$ $\varnothing$. Hence $(y) \alpha_{z} \in Y$. By (P7.3) there exists an $h \in H$ such that $h \alpha_{z}^{-1} \in K_{y}$. Then $(z) \alpha_{z} \notin O_{Z}$, since $I(Z) \cap O_{Z}=\varnothing$. Hence by (P7.1), $h \alpha_{z}^{-1} \notin H_{y}$. Let $h$ and $h^{\prime} \in$ $H_{y}$. Suppose $h h_{y} \alpha_{z}^{-1}=h^{\prime} h_{y} \alpha_{z^{\prime}}^{-1}$ for $z \neq z^{\prime}$. Then $h h_{y}=h^{\prime} h_{y}-\alpha_{z^{\prime}}^{-1} \alpha_{z}$. This contradicts (P7.2). Thus for every element $h \in H_{y}$ there are $|Z|+1$ elements of $K_{y}$, namely $\{h\} \cup\left\{h \alpha_{z}^{-1}: z \in Z\right\}$. Hence $o\left(K_{y}\right)>(|Z|+1) o\left(H_{y}\right)$.

LemMA 8. Let $A$ be a group acting on a set $X$. Let $X=\cup_{i=0}^{s} X_{i}$ be a reducible partition. Suppose $Y=X_{i}, i \geqslant 1$, breaks into at least two orbits under $H$. Let $O$ be an orbit of $H$ on $Y$. Suppose $|O| \geqslant 2$. Then if $y \in O$ and $K=\left\langle H,\left\langle\alpha_{y}: y \in Y\right\rangle\right\rangle$, then $o\left(K_{y}\right)>o\left(H_{y}\right)$.

Proof. Let $y \in O$. Suppose there exists a $y^{*} \in Y-y$ such that $(y) \alpha_{y_{*}} \in O$. By (P7.3) there exists an $h \in H$ such that $h \alpha_{y^{*}}^{-1} \in K_{y}$. Since $\left(y^{*}\right) \alpha_{y^{*}} \notin O$ by (P7.1), $h \alpha_{y^{*}}^{-1} \notin H_{y}$. Hence $o\left(K_{y}\right)>o\left(H_{y}\right)$. Suppose for all $y^{*} \in Y-y,(y) \alpha_{y^{*}} \in O$. Since $|Y|-1>|Y|-|O|$ by the pigeon-hole principle some $y^{-}$is the image of $y$ under $\alpha_{y^{\prime}}^{-1}$ and $\alpha_{y^{\prime \prime}}^{-1}$. Hence $\alpha_{y^{\prime}}^{-1} \alpha_{y^{\prime \prime}} \in K_{k} \backslash H_{y}$. Hence $o\left(K_{y}\right)>o\left(H_{y}\right)$.

Let $A$ be a group acting on a set $X$. Suppose that $X=\cup_{i=0}^{s} X_{i}$ is a reducible partition of $X$. Suppose $Y \subset X_{j}$ for some $j \geqslant 1$. Suppose $H \subseteq A$ and suppose $O_{1}, \ldots, O_{u}$ are the orbits of $H$ where $Y \subset \cup_{k=1}^{u} O_{k}$. Suppose $I(Y) \cap \cup_{k=1}^{u} O_{k}$ $=\varnothing$. Suppose for every $y \in Y$ and $a \in \cup_{k=1}^{u} O_{k}$ that $(a) \alpha_{y} \notin U_{y}$ where $U_{y}$ is the orbit of $H$ containing $(y) \alpha_{y}$. Let

$$
K=\left\langle H,\left\langle\alpha_{z}: z \in Y\right\rangle\right\rangle .
$$

Let $Q_{1}, \ldots, Q_{u^{\prime}}$ be the orbits of $K$. Let $M$ be the pointwise stabilizer of $\cup_{m=1}^{u^{\prime}} Q_{m}$. Let

$$
\begin{aligned}
K^{-} & =K / M, \\
H^{\prime} & =\langle H, M\rangle, \text { and } \\
H^{-} & =H^{\prime} / M .
\end{aligned}
$$

LemMA 9. Let $K$ and $H$ be as in (2.8). Let $K^{-}$and $H^{-}$be as in (2.9). If $f$ is the least integer such that $f o\left(H^{-}\right) \leqslant f$ then $\left[K^{-}: H^{-}\right] \geqslant f$ and $[K: H] \geqslant f$.

Proof. The proof follows the proof of (L4 C2). Suppose $\mathrm{H}^{-}$contains a nontrivial normal subgroup $N^{-}$of $K^{-}$. Since $N^{-}$is nontrivial it acts nontrivially on one of the orbits $Q_{m}, 1 \leqslant m \leqslant u^{\prime}$. However, the orbits of $N$ are blocks of $K$ on $Q_{m}$. By assumption, there exists a $k$ such that $Q_{m} \supset O_{k}$. Hence $Q_{m} \cap Y \neq \varnothing$. Since $N$ has nontrivial orbits on $Q_{m}$ there exists a nontrivial orbit $O$ of $N$ containing $y \in O_{s} \cap Y$. Hence there exists an element $n \in N$ containing a cycle $(y a \cdots)$ where $a \in O_{k}$. The cycle $\left((y) \alpha_{y}(a) \alpha_{y} \cdots\right)$ is contained by $\alpha_{y}^{-1} n \alpha_{y}$. By definition (y) $\alpha_{y} \in U$ and $(a) \alpha_{y} \notin U_{y}$. Since $\alpha_{y}^{-1} n \alpha_{y} \in N \subset H^{-}$a contradiction follows. As in $(\mathrm{L} 4 \mathrm{Cl}) \mathrm{H}^{-}$containing no nontrivial normal subgroup of $K^{-}$means that $o\left(K^{-}\right) \mid\left[K^{-}: H^{-}\right]$!. Hence $\left[K^{-}: H^{-}\right] \geqslant f$. By the correspondence theorem $\left[K: H^{\prime}\right] \geqslant f$. 
Let $A$ be a group acting on a set $X$. Let $X=\cup_{i=0}^{s} X_{i}$ be a reducible partition. Let $H=\left\langle\alpha_{z}: z \in \cup_{i=r}^{s} X_{i}\right\rangle$. Suppose $H$ has reducing orbits $O_{1}, \ldots, O_{p}$ such that $X_{s} \cap O_{v} \neq \varnothing$ for $1 \leqslant v \leqslant p$. For $1 \leqslant u \leqslant p$ and $1<k<r-s+1$ let

$$
H_{u k}=\left\langle\alpha_{z}: z \in\left(\bigcup_{v=1}^{u-1} \bigcup_{m=r}^{s} O_{v} \cap X_{m}\right) \cup\left(\bigcup_{m=s-(k-1)}^{s} O_{u} \cap X_{m}\right)\right\rangle .
$$

If $k>1$ then let $H_{u k-1}$ have reducing orbits $O_{1}^{-}, \ldots, O_{c}^{-}$with $\cup_{w=1}^{c} O_{w}^{-} \subseteq O_{u}$. Let $H_{u k}$ act on reducing orbits $Q_{1}, \ldots, Q_{d}$ with $\cup_{e=1}^{d} Q_{e} \subseteq O_{u}$. Let $N_{u k}$ be the pointwise stabilizer of $\cup_{e=1}^{d} Q_{e}$. Let

$$
\begin{aligned}
H_{u k}^{-} & =H_{u k} / N_{u k}, \\
H_{u k-1}^{\prime} & =\left\langle H_{u k-1}, N_{u k}\right\rangle, \quad \text { and } \\
H_{u k-1}^{-\prime} & =H_{u k-1}^{\prime} / N_{u k} .
\end{aligned}
$$

Corollary 9.1. Let $A$ be a group acting on a set $X$. Let $X=\cup_{i=0}^{s} X_{i}$ be a reducible partition. For $1 \leqslant u \leqslant p$ and $1 \leqslant k \leqslant r-s+1$ let $H_{u k}$ be defined in (2.10). If $u=1$ and $k=1$ then $o\left(H_{11}\right)>\left(\left|X_{s} \cap O_{1}\right|+1\right)$. If $u>1$ and $k=1$ then $o\left(H_{u 1}\right) \geqslant\left(\left|X_{s} \cap O_{s}\right|+1\right) o\left(H_{u-1 s-r+1}\right)$. Let $H_{u k}^{-}$and $H_{u k}^{-1}$ be defined in (2.11). Let $f_{u k}$ be the least integer such that $f_{u k} ! \geqslant f_{u k} o\left(H_{u k-1}^{-1}\right)$, where for $3<k<s-r+1$ and $1<u \leqslant p, f_{u k}>f_{u k-1}$ and $f_{u 2} \geqslant 3$. Then (1) $\left[H_{u k}^{-}: H_{u k-1}^{-\prime}\right] \geqslant f_{u k},\left[H_{u k}: H_{u k-1}\right]>$ $f_{u k}$, and $o\left(H_{u 2}^{-}\right) \geqslant 6\left|X_{s} \cap O_{u}\right|$. (2) $o\left(H_{u k-1}\right) \geqslant f_{u m^{\prime}} \cdots f_{u n} o\left(H_{u n-1}\right)$ where $1<u<p$ and $2 \leqslant n<m^{\prime} \leqslant s-r+1$. (3) $o(H)>\Pi_{v=1}^{p}\left(\Pi_{k=2}^{s-r+1} f_{v k}\left(\left|X_{s} \cap O_{v}\right|+1\right)\right)$.

Proof. Suppose $u=1$ and $k=1$. Then $o\left(H_{11}\right)>\left|X_{s} \cap O_{1}\right|+1$ by (L3). Suppose $u>1$ and $k=1$. By $(\mathrm{L} 1), o\left(H_{u 1}\right)>\left(\left|X_{s} \cap O_{u}\right|+1\right) o\left(H_{u-1 s-r+1}\right)$. Suppose $k>1$. Then $H_{u k}^{-}$and $H_{u k-1}^{-\prime}$ satisfy the hypothesis of (L9). Hence $\left[H_{u k}^{-}: H_{u k-1}^{-1}\right]>$ $f_{u k}$ and by the correspondence theorem $\left[H_{u k}: H_{u k-1}\right]>f_{u k}$.

Suppose for some $3 \leqslant k \leqslant s-r+1$ and $1 \leqslant u<p, f_{u k}<f_{u k-1}$. By definition $o\left(H_{u k-1}^{-}\right) \geqslant f_{u k-1} o\left(H_{u k-2}^{-\prime}\right)$. Hence $\left(f_{u k-1}-1\right) !>o\left(H_{u k-1}^{-\prime}\right)$ since $f_{u k} o\left(H_{u k-1}^{-\prime}\right)<f_{u k}$ ! and $f_{u k}<f_{u k-1}$. To obtain a contradiction the proof proceeds as in (L6) with the following elaboration of the calculation made in (2.3). It is necessary to show that $H_{u k-1} \cap N_{u k} \subseteq H_{u k-1} \cap N_{u k-1}$. If $\varphi \in H_{u k-1} \cap N_{u k}$ then $\varphi$ fixes every point of $\cup_{e=1}^{d} Q_{e}$. However $\cup_{e=1}^{d} Q_{e} \supset \cup_{w=1}^{c} O_{w}^{-}$. Since $\varphi \in H_{u k-1}$ and $\varphi$ fixes every point of $\cup_{w=1}^{c} O_{w}^{-}, \varphi \in H_{u k-1} \cap N_{u k-1}$ and the result holds.

Since $o\left(H_{u 1}^{-{ }^{\prime}}\right) \geqslant 2, f_{u 2} \geqslant 3$. Repeated application of $o\left(H_{u k}\right)>f_{u k} o\left(H_{u k-1}\right)$ gives $o\left(H_{u m^{\prime}}\right) \geqslant f_{u m^{\prime}} \cdots f_{u n} o\left(H_{u n-1}\right)$ where $1<u<p$ and $2<n<m^{\prime}<s-r+1$. By the argument of (L6), $o\left(H_{u 2}^{-}\right) \geqslant 6\left|X_{s} \cap O_{u}\right|$ for $1<u<p$. In summary $o(H)>$ $\Pi_{v=1}^{p}\left(\Pi_{k=2}^{s-r+1} f_{v k}\left(\left|X_{s} \cap O_{v}\right|+1\right)\right)$.

Let $A$ be a group acting on a set $X$. Let $X=\cup_{i=0}^{s} X_{i}$ be a reducible partition of $X$. Let $H \subseteq A$. Let $O_{1}$ and $O_{2}$ be two orbits of $H$ such that $O_{1} \subset \cup_{i=1}^{r+1} X_{i}$ where $0<r<s$, and $O_{2} \subset \cup_{i=r+1}^{s} X_{i}$ where $O_{2} \cap X_{r+1} \neq \varnothing$. Suppose there exists a $z \in O_{2} \cap x_{r+1}$ such that $(z) \alpha_{z} \in O_{1}$. Let $K_{0}=H$ and let $Q_{0}=O$. For $1<j<v$ let $K_{i}=\left\langle K_{i-1}, \alpha_{z_{i}}\right\rangle$ where $z_{i} \in Q_{i-1} \cap X_{r+1}$ and $\alpha_{z_{i}} \notin K_{i}$ and let $Q_{i}$ be the orbit of $K_{i}$ containing $Q_{i-1}$. Since $A$ is finite, $u$ is bounded. Let $K=K_{u}$ and $Q=Q_{u} . K$ is termed an $O_{1}$ extension of $H$. 
Corollary 9.2. Suppose $A$ is a group acting on a set $X$. Suppose $X=\cup_{i=0}^{s} X_{i}$ is a reducible partition. Let $H \subseteq A$ with orbits $O_{1} \subset \cup_{i=1}^{r+1} X_{i}$ where $0<r<s$ and $\mathrm{O}_{2} \subset \cup_{i=r+1}^{s} X_{i}$ with $\mathrm{O}_{2} \cap X_{r+1} \neq \varnothing$. Let $\mathrm{K}$ be an $\mathrm{O}_{1}$ extension of $\mathrm{H}$. Let $N=K_{Q}$ and $H^{\prime}=\langle H, N\rangle$. Let $K^{-}=K / N$ and $H^{-\prime}=H^{\prime} / N$. Then if $a$ is the least integer such that $a ! \geqslant a o\left(H^{-\prime}\right)$ then $[K: H] \geqslant a$.

Proof. This is a direct consequence of ( $\mathrm{L4} \mathrm{C1})$.

\section{A lower bound for $o\left(A_{x}\right)$.}

TheOREM 1. Let $A$ be a group acting on a set $X$. Let $X=\cup_{i=0}^{t} X_{i}$ be a reducible partition of $X$. Then there exists an $x \in X$ such that $o\left(A_{x}\right)>t$ !.

Proof. The case $t=1$ is clear. Let $t=2$. Let $H=\left\langle\alpha_{z}: z \in X_{2} \cup X_{1}\right\rangle$. Suppose $H$ acts on a single orbit $O=X_{0} \cup X_{1} \cup X_{2}$. By (L3 Cl), $H$ is not regular. Hence for $x \in O, o\left(A_{x}\right) \geqslant 2$.

Let $t \geqslant 3$. Suppose $\left\{X_{i}\right\}_{i=1}^{t-1}$ are singletons. Let $u=\left|X_{t}\right|$. Let $H_{j}=\left\langle\alpha_{z}: z \in X_{t}\right.$ $\left.\cup \cdots \cup X_{t-(j-1)}\right\rangle, \quad 1 \leqslant j \leqslant t-1$. By (L3), $o\left(H_{1}\right)>u+1$. Since $H_{j}$ fixes $X_{t-(j+1)}, o\left(H_{j+1}\right) \geqslant(j+1+u) o\left(H_{j}\right), 1<i \leqslant t-2$. Hence

$$
o\left(H_{t-1}\right) \geqslant(t-1+u) o\left(H_{t-2}\right) \text {. }
$$

Hence $o\left(H_{t-1}\right) \geqslant(t-1+u) \cdots(u+2)(u+1)$. Let $x_{1} \in X_{1}$. Since $\cup_{i=1}^{t} X_{i}$ is an orbit of $H_{t-1}$ and $\left|\cup{ }_{i=1}^{t} X_{i}\right|=u+t-1$, by (P7.4),

$$
o\left(A_{x_{1}}\right) \geqslant o\left(H_{t-1}\right) /(t+u-1) \geqslant(t+u-2) \cdots(u+1) .
$$

Since $\alpha_{x_{1}}$ fixes $x_{2}$ by $(\mathrm{L7} \mathrm{C} 1), o\left(A_{x_{2}}\right) \geqslant 2 \cdot(t+u-2) \cdots(u+1) 2 \geqslant t$ ! provided $u \geqslant 2$. If $u=1$ let $H=\left\langle\alpha_{z}: z \in \cup{ }_{i=1}^{t-1} X_{i}\right\rangle$. Since $x_{t}$ is fixed by $H, o\left(A_{x_{t}}\right)>o(H)$. However, $o(H) \geqslant t$ ! by (L6).

Suppose not all of the $\left\{X_{i}\right\}_{i=1}^{t-1}$ are singletons. Suppose $t>4$. Let $\left|X_{t}\right|<t+1$. Suppose $\left|X_{t-1}\right| \geqslant 2$. Let $H_{1}=\left\langle\alpha_{z}: z \in X_{t-1}\right\rangle$ and $H_{2}=\left\langle\alpha_{z}: z \in X_{t-1} \cup X_{t-2}\right\rangle$. If $H_{3}$ reduces $X_{t-2}$, in addition $o\left(H_{3}\right) \geqslant 120$ by (L6 C4). Let $H_{t-1}=\left\langle\alpha_{z}: z \in\right.$ $\left.\cup \underset{i=1}{t-1} X_{i}\right\rangle$. By (L6 C4), $o\left(H_{t-1}\right) \geqslant(t+1)$ !. Since $H_{t-2} \subseteq A_{\left(X_{t}\right)}$ and $\left|X_{t}\right|<t+1$, $o\left(A_{x_{t}}\right) \geqslant t$ !, where $x_{t} \in X_{t}$.

Suppose $\left|X_{t-1}\right|=1$. Suppose $t \geqslant 5$. The arguments just applied to the case $\left|X_{t-1}\right| \geqslant 2$ show that if $H_{t-2}=\left\langle\alpha_{z}: z \in \cup_{i=1}^{t-2} X_{i}\right\rangle$ is generated reducing from the top then $o\left(H_{t-2}\right) \geqslant t$ !. As $H_{t-2} \subseteq A_{x_{t-1}}$ this implies $o\left(A_{x_{t-1}}\right) \geqslant t$ !. Hence suppose $\left|X_{t-2}\right|=1$ if $t \geqslant 5$.

Suppose $\left|X_{1}\right| \geqslant\left|X_{t}\right|$. If $\left|X_{1}\right|=1$ then $\left|X_{t}\right|=1$ and the result follows by (L6).

Suppose $\left|X_{1}\right| \geqslant 2$. Let $H_{t-1}=\left\langle\alpha_{z}: z \in \cup_{i=1}^{t-1} X_{i}\right\rangle$. By (L6), $o\left(H_{t-1}\right) \geqslant t !\left|X_{1}\right|$ and the result follows using $H_{t-1} \subseteq A_{\left(X_{t}\right)}$.

Suppose $\left|X_{t}\right|>\left|X_{1}\right|$. By the above argument $\left|X_{t-1}\right|=1$. Let $\left|X_{t}\right|=2$. Then $H_{1} \cong S_{3}$. If $H_{t-2}=\left\langle\alpha_{z}: z \in \cup_{i=3}^{t} X_{i}\right\rangle$, then by (L6 C4), $o\left(H_{t-2}\right)>t$ !. Since $\left|X_{1}\right|=1$ the result follows.

Suppose $\left|X_{1}\right|=1$ and $\left|X_{t}\right| \geqslant 3$. By (L6 C4), $o\left(H_{3}\right)>5$ ! and so if $t>5$ then $o\left(H_{t-2}\right) \geqslant t$ ! by (L6 C4). Hence $o\left(A_{x_{1}}\right) \geqslant t$ ! since $H_{t-2} \subset A_{x_{1}}$. If $t=4$ then $o\left(H_{2}\right)$ $\geqslant 20$ by (L3) and (LA C2). Since $o\left(\left(H_{3}\right)_{x_{3}}\right)=o\left(\left(H_{3}\right)_{x_{1}}\right)>o\left(H_{2}\right)>20$ and $x_{3}$ is fixed by $\alpha_{x_{1}}, o\left(A_{x_{3}}\right) \geqslant 4$ !. 
Suppose $\left|X_{t}\right| \geqslant 3$ and $\left|X_{1}\right|>1$. Let $\left|X_{t}\right|=3$. If $t \geqslant 5$ then by (L6 C4), $o\left(H_{t-2}\right)>$ $t$ !. If $X_{1}$ is an orbit of $H_{t-2}$ then $o\left(A_{x_{1}}\right) \geqslant t$ ! by (L7). If $\left|X_{1}\right|$ is not an orbit by (P7.4), $o\left(A_{x_{1}}\right) \geqslant 2 t ! /\left|X_{1}\right|$. Note that $\left|X_{1}\right|<2$ since $\left|X_{t}\right|>\left|X_{1}\right|$. If $t=4$ then if $H_{2}=\left\langle\alpha_{z}: z \in X_{1} \cup X_{2}\right\rangle, o\left(H_{2}\right) \geqslant 12$ by (L6). Let $H_{3}=\left\langle\alpha_{z}: z \in X_{1} \cup X_{2} \cup\right.$ $X_{4}>. H_{2}$ fixes $x_{3}$ so $o\left(\left(H_{3}\right)_{x_{4}}\right)=o\left(\left(H_{3}\right)_{x_{3}}\right) \geqslant 12$. By $(\mathrm{L7Cl}), o\left(\left(H_{4}\right)_{x_{4}}\right)>24$. Let $\left|X_{t}\right|=4,5$, or 6. By (L3), $o\left(H_{1}\right) \geqslant 2\left|X_{t}\right|$. By $(\mathrm{L6} \mathrm{Cl}), o\left(H_{t-2}\right) \geqslant(t+1) \cdots 5 \cdot$ $2\left|X_{t}\right|$. By (L8),

$$
\begin{aligned}
o\left(A_{x_{1}}\right) & \geqslant 2 o\left(H_{t-2}\right) /\left|X_{1}\right| \geqslant 2 o\left(H_{t-2}\right) /\left(\left|X_{t}\right|-1\right) \\
& \geqslant 2(t+1) \cdots 5 \cdot 2\left|X_{t}\right|\left(\left|X_{t}\right|-1\right) \geqslant t ! .
\end{aligned}
$$

Let $\left|X_{t}\right| \geqslant 7$. Since $t \geqslant 6$ as $\left|X_{t}\right| \leqslant t+1,\left|X_{t-2}\right|=1$. By (L3), $o\left(H_{1}\right) \geqslant\left|X_{t}\right|+1 \geqslant$ 8. Since $x_{t-2}$ is fixed by $H_{1}, o\left(H_{2}\right) \geqslant 9\left(\left|X_{t}\right|+1\right) \geqslant 72$. By (L6), $o\left(H_{t-2}\right) \geqslant$ $(t+1) \cdots 6 \cdot 9\left(\left|X_{t}\right|+1\right)$. By (L8),

$$
\begin{aligned}
o\left(A_{x_{1}}\right) & \geqslant 2 o\left(H_{t-2}\right) /\left|X_{1}\right| \geqslant 2 o\left(H_{t-2}\right) /\left|X_{1}\right| \\
& \geqslant 2(t+1) \cdots 6 \cdot 9\left(\left|X_{t}\right|+1\right) /\left|X_{1}\right| \geqslant t ! .
\end{aligned}
$$

Let $\left|X_{t}\right| \geqslant t+2$ and let $\left|X_{1}\right| \geqslant \frac{1}{2}\left|X_{t}\right|$. Let $H_{t}=\left\langle\alpha_{z}: z \in \cup_{i=1}^{t-1} X_{i}\right\rangle$ be generated reducing from the bottom. By (L6), $o\left(H_{t-1}\right) \geqslant t !\left|X_{1}\right|$. Since $X_{t}$ is not a singleton (L5) gives $o\left(A_{x_{t}}\right) \geqslant 2 o\left(H_{t-1}\right) /\left|X_{t}\right| \geqslant t ! 2\left|X_{1}\right| /\left|X_{t}\right| \geqslant t$ !.

Suppose $\left|X_{1}\right|<\frac{1}{2}\left|X_{t}\right|$. Let $H_{t-2}=\left\langle\alpha_{z}: z \in \cup_{i=3}^{t} X_{i}\right\rangle$. Suppose $t>4$. Hence $\left|X_{t}\right| \geqslant 7$ as $\left|X_{t}\right| \geqslant t+2$. By (L3), $o\left(H_{1}\right) \geqslant 8$. By (L6 C3), $o\left(H_{t-2}\right) \geqslant$ $(t+1) \cdots 5\left|X_{t}\right| \geqslant(t+1) \cdots 5 \cdot 2\left|X_{1}\right|$. If $X_{1}$ is a singleton, then $o\left(A_{x_{1}}\right)>$ $o\left(H_{t-2}\right) \geqslant(t+1) \cdot \cdots 5 \cdot 8 \geqslant t$ !. If not, apply (L8) to obtain $o\left(A_{x_{1}}\right)>$ $2 o\left(H_{t-2}\right) /\left|X_{1}\right| \geqslant t$ !. Let $t=4$ and let $\left|X_{4}\right|=6$. By (L3), $o\left(H_{1}\right) \geqslant 12$. By (L6 C3), $o\left(H_{2}\right) \geqslant 5 o\left(H_{1}\right)$. If $\left|X_{4}\right| \geqslant 7, o\left(H_{1}\right) \geqslant 8$ by (L3). By (L6 C3), $o\left(H_{2}\right)>5 o\left(H_{1}\right) \geqslant$ 40. If $X_{1}$ is a singleton, the result follows. Suppose $X_{1}$ is not a singleton. Let $\left|X_{4}\right|=6$. By (L8), $o\left(A_{x_{1}}\right) \geqslant 2 \cdot 5 o\left(H_{1}\right) /\left|X_{1}\right| \geqslant 20\left|X_{4}\right| /\left|X_{1}\right| \geqslant 40$. Let $\left|X_{4}\right|=7$. By (L6 C3), $o\left(H_{2}\right) \geqslant 40$. By (L8), $o\left(A_{x_{1}}\right) \geqslant(2 \cdot 40) / 3>4$ !. Let $8<\left|X_{4}\right|<14$. By (L3) and (L6 C3), $o\left(H_{2}\right) \geqslant 5 \cdot 2\left|X_{4}\right|$. By (L8), $o\left(A_{x_{1}}\right) \geqslant 2 \cdot 5 \cdot 2\left|X_{4}\right| /\left|X_{1}\right|>40$. Let $\left|X_{4}\right|$ $=15$. By (L3) and (L6 C3), $o\left(H_{2}\right) \geqslant 7 \cdot 16$. By (L8), $o\left(A_{x_{1}}\right)>2 \cdot 7 \cdot 2\left|X_{4}\right| /\left|X_{1}\right|>$ 24. Let $\left|X_{4}\right| \geqslant 16$. By (L3), $o\left(H_{1}\right)>24$. By (L6 C3), $o\left(H_{2}\right)>6\left|X_{4}\right|$. By (L8), $o\left(A_{x_{1}}\right) \geqslant 2 \cdot 6 \cdot 2\left|X_{1}\right| /\left|X_{1}\right|=24$ !

Let $t=3$. The proposition has been shown in the case $X_{1}$ and $X_{2}$ are singletons. Suppose $\left|X_{3}\right| \leqslant 4$. Let $H_{1}=\left\langle\alpha_{z}: z \in X_{2}\right\rangle$ and $H_{2}=\left\langle\alpha_{z}: z \in X_{2} \cup X_{1}\right\rangle$. If $X_{3}$ is a singleton then $o\left(\left(H_{2}\right)_{x_{3}}\right)>3$ ! by (L6). Suppose $X_{3}$ is not a singleton. Suppose $\left|X_{2}\right| \geqslant 2$. By (L6 C2), $o\left(H_{2}\right) \geqslant 20$. By (L5), $o\left(A_{x_{3}}\right) \geqslant 2 o\left(H_{2}\right) /\left|X_{3}\right| \geqslant 20 / 2 \geqslant 10$. Suppose $X_{2}$ is a singleton and $X_{1}$ is not one. Let $H_{1}=\left\langle\alpha_{z}: z \in X_{1}\right\rangle$. By (L6), $o\left(H_{2}\right) \geqslant 6\left|X_{1}\right|$. By (L5),

$$
o\left(A_{x_{3}}\right) \geqslant 2 o\left(H_{2}\right) /\left|X_{3}\right| \geqslant 6 \cdot 2\left|X_{1}\right| / 4 \geqslant 6 .
$$

Let $\left|X_{3}\right| \geqslant 5$. Suppose $2\left|X_{1}\right| \geqslant\left|X_{3}\right|$. Let $H_{2}=\left\langle\alpha_{z}: z \in X_{1} \cup X_{2}\right\rangle$. By (L6), $o\left(H_{2}\right) \geqslant 6\left|X_{1}\right|$. By assumption, $\left|X_{3}\right| \geqslant 5$. By (L5),

$$
o\left(\left(H_{3}\right)_{x_{3}}\right) \geq \frac{2 o\left(H_{2}\right)}{\left|X_{3}\right|}>6 .
$$


Suppose $\left|X_{3}\right| \geqslant 2\left|X_{1}\right|$. Let $H_{1}=\left\langle\alpha_{z}: z \in X_{3}\right\rangle$. If $X_{1}$ is a singleton, then since $o\left(H_{1}\right) \geqslant 8$, by (L3) the result follows. If $X_{1}$ is a doubleton then by (L8), $o\left(A_{x_{1}}\right)>$ $2 o\left(H_{1}\right) / 2 \geqslant 8$. Suppose $\left|X_{1}\right| \geqslant 3$. If $X_{1}$ splits into 3 orbits under $H_{1}$ then there exists an orbit $O \subseteq X_{1}$, with $3|O| \leqslant\left|X_{1}\right|$. Hence for $x_{1} \in O$, $O\left(\left(H_{1}\right)_{x_{1}}\right)>$ $o\left(H_{1}\right) /|O| \geqslant\left(\left|X_{3}\right|+1\right) /|O| \geqslant 2\left|X_{1}\right| /|O| \geqslant 6$. Suppose $X_{1}$ splits into 2 orbits. If either orbit is a singleton, then $o\left(A_{x_{1}}\right) \geqslant o\left(\left(H_{1}\right)_{x_{1}}\right)>8$. Suppose neither is a singleton. Suppose only one of the orbits of $H_{1}$ on $X_{1}$ is part of a reducing orbit of $\mathrm{H}_{2}$ or both are part of different reducing orbits. Let $\mathrm{O}$ be an orbit such that $2|O| \leqslant\left|X_{1}\right|$. Either $O$ is part of the reducing orbit of $H_{2}$ or it is stabilized by $H_{2}$. Since $|O| \geqslant 2$ and $\alpha_{x_{2}}^{-1}$ sends all but at most one element of $O$ into $O$ for $x_{2} \in X_{2}$, there exists an $x_{1} \in O$ such that $\left(x_{1}\right) \alpha_{x_{2}}^{-1} \in O$ for some $x_{2} \in X_{2}$. By (P7.3) and (P7.1), there exists a $\psi \in H_{1}$ such that $\alpha_{x_{2}}^{-1} \psi^{-1} \notin\left(H_{1}\right)_{x_{1}}$. However

$$
o\left(\left(H_{1}\right)_{x_{1}}\right) \geqslant O\left(H_{1}\right) /|O| \geqslant\left(\left|X_{3}\right|+1\right) /|O| \geqslant 2 \cdot 2 \cdot\left(\left|X_{1}\right|+1\right) /\left|X_{1}\right|>4 .
$$

Hence $o\left(A_{x_{1}}\right) \geqslant 8$. Suppose both orbits are part of a single reducing orbit of $\boldsymbol{H}$. Since $o\left(\left(H_{1}\right)_{x_{4}}\right) \geqslant 4$ for $x_{1} \in O, o\left(\left(H_{2}\right)_{x_{3}}\right)>4$. Since $X_{3}$ is part of a single reducing orbit of $H_{2}$ and if $H_{3}=\left\langle\alpha_{z}: z \in \cup \bigcup_{i=0}^{3} X_{i}\right\rangle$ then $o\left(\left(H_{3}\right)_{x_{3}}\right) \geqslant 2 o\left(\left(H_{2}\right)_{x_{3}}\right)>8$ by (L7 C1).

Suppose $X_{1}$ is a single orbit under $H_{1}$. Since $o\left(H_{1}\right) \geqslant\left|X_{3}\right|+1 \geqslant 2\left|X_{1}\right|, o\left(\left(H_{1}\right)_{x_{1}}\right)$ $\geqslant 2$. Since $X_{1}$ is not a singleton, $o\left(\left(H_{2}\right)_{x_{1}}\right) \geqslant 4$ by (L7). Since $X_{3}$ is part of a single reducing orbit of $\mathrm{H}_{2}, o\left(\left(\mathrm{H}_{2}\right)_{x_{3}}\right) \geqslant 4$. By $(\mathrm{L} 7 \mathrm{Cl}), o\left(\left(\mathrm{H}_{3}\right)_{x_{3}}\right)>8$.

\section{BIBLIOGRAPHY}

1. Neil Robertson and J. A. Zimmer, Automorphisms of subgraphs obtained by deleting a pendant vertex, J. Combin. Theory Ser. B 12 (1972), 169-172.

2. W. T. Tutte, A family of cubical graphs, Proc. Cambridge Philos. Soc. 43 (1948), 459-474.

Department of Mathematics, Louisiana State University, Baton Rouge, Louisiana 70803

Department of Mathematics, Ohio State University, Columbus, Oho 43210 